\title{
The Key Technology Research of Virtual Laboratory based On Cloud Computing
}

\author{
Ling Zhang \\ Nanjing Communications Institute of Technology, Nanjing 211188, China
}

\begin{abstract}
Keywords: Cloud Computing, Training Cloud, Virtualization, Load balancing, VM Dynamic Migration
\end{abstract}

\begin{abstract}
The emergence of cloud computing to higher vocational education has brought new changes, based on cloud Desktop Network virtualization technology can make the traditional training model has been tremendous innovation, cloud desktop virtualization technology can be used to carry out a wide range of online virtual training teaching mode, from the true sense, training clouds can open laboratory, effectively alleviate the shortage of equipment pressure, and reduce maintenance strength, effectively improve the existing experimental teaching system. Based on the practical cloud as foothold, in view of the virtualization technology research, analyses the design of virtualized computing resources pool, the realization of the virtual machine live migration ability, etc, are discussed, trying to realize system's high availability and load balancing. And hope to provide some intends to explore and ideas for further research work.
\end{abstract}

\section{Training key technology analysis of training cloud platform}

Training Cloud is a cloud computing environment in higher education typical of a specific application, through a cloud environment can provide students with training related platform services, resources, services, and process services. Training cloud platform involves two core technologies: Virtualization and load balancing, these two technologies to ensure reliable operation and dynamic expansion of the cloud platform.

Training virtualization technology is the foundation of the cloud, mainly refers to computer and operating system platform for virtualization, including virtual server, CPU virtualization, virtual memory, and storage virtualization. The so-called virtualization refers to the logical representation of computational physics resources, and out of the shackles of the physical media. Virtualization technology can simplify access to computing resources and management, using common standard user interfaces and convenient access to system resources, replacing physical access to these resources to their implementation. Virtualization technology is implemented in cloud computing resource sharing of the core technology. Through virtualization, users can easily access the server and storage resources, without having to specify a specific server and storage resources. For data centers, virtualization technology to solve the dynamic data center resource management issues. At the same time, virtualization technology is very good to solve the problem of heterogeneous resources, it is possible to achieve real independence platform. The system load balancing problem can be dynamically adjusted by remapping the virtual machine to a physical machine. Due to these advantages of virtualization technology, Cloud computing using the virtualization technology to a single physical server virtualization resources out several high performance general performance of the virtual machine, in order to meet the needs of users.

As cloud computing is another key technology, in this article, the description for load balancing, is through the training load redistribution of cloud nodes to get more system resources and improve the efficiency of the task is completed, Under the premise of minimizing the user requests time and overhead, to maximize system throughput and effectively reduce server overload, meanwhile, requirements for system quality of service, reliability and scalability to provide effective protection. Through the following two aspects to ensure the content load balancing to achieve: one is that based on task distribution of ideas, in the deployment phase of large-scale data stream or submit concurrent access to multiple servers on demand parallel processing; the second is based on dynamic migration of ideas, reschedule single individual heavy high load server, the overrun task 
migration to another server for processing.

\section{Virtualization Model of Training cloud platform}

Generally, we can put a cloud-based training system is technically classified as a four-layer structure: Service-Oriented Architecture (SOA) build layer, management middleware layer, resource pool layer, physical resource layer. SOA build layer is directly oriented applications, package cloud computing capability as a standard Web Services; management middleware layer service to SOA build layer, provide the necessary support of cloud computing resources and management; resource pool layer USES the underlying hardware virtualization technology integration system, forming a virtualized resource pool, includes various hardware resources, operating systems, database systems, and a collection of applications; physical resource layer involves all the hardware resources, including server hardware systems, network hardware systems. Relying on virtualization technology, after the training room in a variety of resources to achieve the future of virtualization, the traditional physical host becomes virtual machines in computing resources pool, users use telnet client terminal to complete action on the virtual machine, receive service support computer resource pool. Involved in related operations, calculations were carried out on high-performance servers, with virtualization technology to realize the centralized control of the training room maintenance management, management changes from the original physical machine to manage virtual machines. Through the training room of the cloud environment virtualized computing resource allocation, scheduling, management and other aspects of the analysis, formed the following virtualized computing resource pool model.

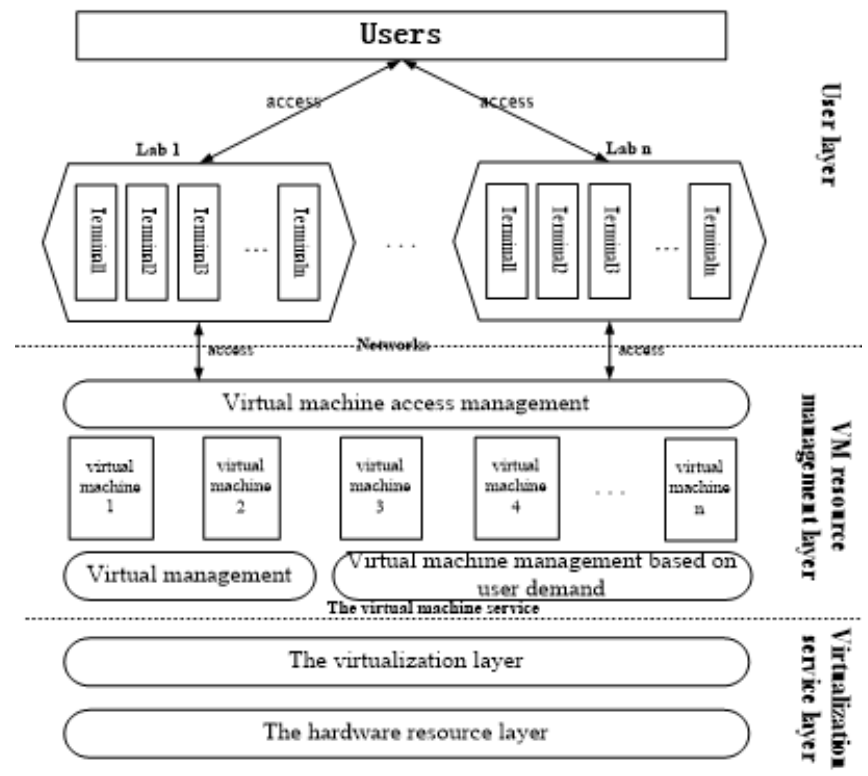

Fig.1: The model of calculating resource pool

User application layer: this layer user uses the client terminal remotely log into the virtual machine to operate, directly facing specific application to expand the experimental task, you can use diskless workstations, thin clients as a client.

Virtual machine resource management: consists of virtual machines and related management systems. This layer, based on the virtual hardware resources to meet the huge demand of the virtual machine will be created, under the management system to control concurrent processing data. At the same time, this layer bears on system virtualization management, responsible for selecting the appropriate virtual machine template generation and destroy virtual machine basis for the demand; establish the mapping between the user terminal and the virtual machine, realize the user access to a virtual machine; solve the load balancing between servers, in order to meet the needs of students' virtual training. According to the curriculum schedule of the course, time, location, class, number and other relevant factors, automatically generated to meet the needs of the virtual machine configuration when preparation time before class, the virtual machine is automatically destroyed 
after school. Thus, to achieve the needs of different courses, meet the practical Teaching needs of different courses.

Virtual hardware resource layer: consists of hardware resources and virtualization layer, which operator, a storage device, network equipment and other resources constitute the hardware resource layer; the virtualization layer provides the runtime environment and resources on one virtual machine resource management, it use virtualization layer abstract technology complete virtual abstraction of the hardware resource layer, so that the operating system and hardware resources separated, By Virtual Machine Monitor(VMM) performed on the dispatching operation system in the physical hardware resources, and to provide a full range of virtual hardware interface. At the same time, solves in a large number of virtual machines cloud server and multiple operating systems to run independently concurrency issues.

\section{Load balancing under the virtualized environments}

\section{Dynamic migration conditions of virtual machine.}

Dynamic migration of virtual machines need to solve many problems, mainly related cpu migration, memory migration, storage migration and other equipment migration. Because of the virtual machine live migration process memory data amount is larger, so the migration difficulty is higher; therefore, the most complicated part is the memory migration in the dynamic migration of virtual machines. Analysis to obtain the dynamic migration parameters are as follows: (1) Minimal interruption. Because downtime service can not be performed, so dynamic migration process to try to minimize downtime.(2) Consistency. During the migration process, source and destination hosts must be synchronized; otherwise, it will affect the transition effect and stability. (3) Minimal interference. To protect the virtual machine during the migration process, interfere with the implementation of other services. (4)Transparency. During the migration process, migrations both safeguard all network connections and applications, maintaining the original state does not change.

\section{Dynamic migration framework.}

This paper studies the virtual machine live migration of overall framework consists with migration run module, migration freeze module, migration listening module and wake the target domain module the interaction between several modules, function influence each other, common to complete the virtual machine live migration process.

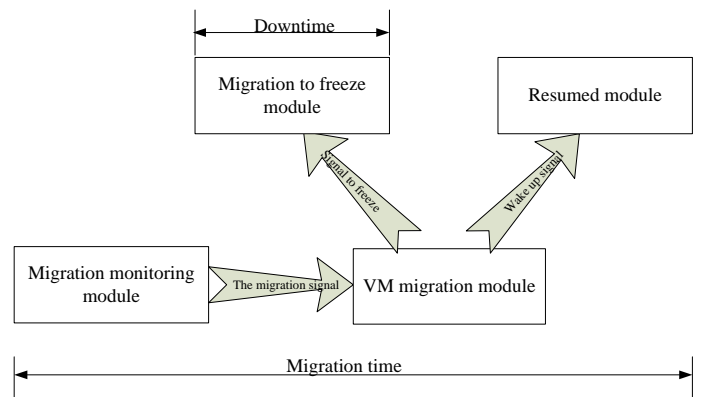

Fig.2:The framework of VM live migration

Migration listener module: the main function of this module is to start the migration; the main work is to determine the migrated objects, when to migrate and where to migrate. Virtual Machine Monitor (VMM) is responsible for managing all running Virtual Machines, if the operating system CPU utilization is too high or low memory, the need for virtual machine migration, it will send a request to the VMM migration of signals, VMM on the managed device resource utilization, virtual machine operation analysis to determine which virtual machines to determine the migration, and accepted by which devices are migrated virtual machine, all the decision-making information to form a package to package submitted to the system, the system according to actual completion of follow-up.

Migration run modules: this module is primarily responsible for implementation of the migration process. This module is mainly completed work is as follows (see figure 3 for the specific process migration steps): the first step, migration listener module issues allow "Migration signal", 
meanwhile, collect migrate domain Q and the operation of the related domain information, and to encapsulate collected information. The second step is freeze migration domain Q. The third step, enter the stage of "iterative pre copy". The fourth step is to enter the "copy down" phase. The fifth step, the copy is completed. The sixth step, activate the target domain. Operation Migration is not only related to the module's total consumption of time and downtime, but also directly affects the performance of the target domain after the migration is complete.

Migration freeze modules: this module is the solution to freeze when migrating domain $\mathrm{Q}$, as well as how to ensure the quality of service of the original system does not change after the freeze, the "continuity" of user services. Achieve migrating domain Q and the target domain D in sync.

Wake target module: this module is used to determine the target domain wake-up time, and settling time a new virtual machine. But also to protect the wake-up, the migration field Q and the target domain D services in sync.

Dynamic migration process.

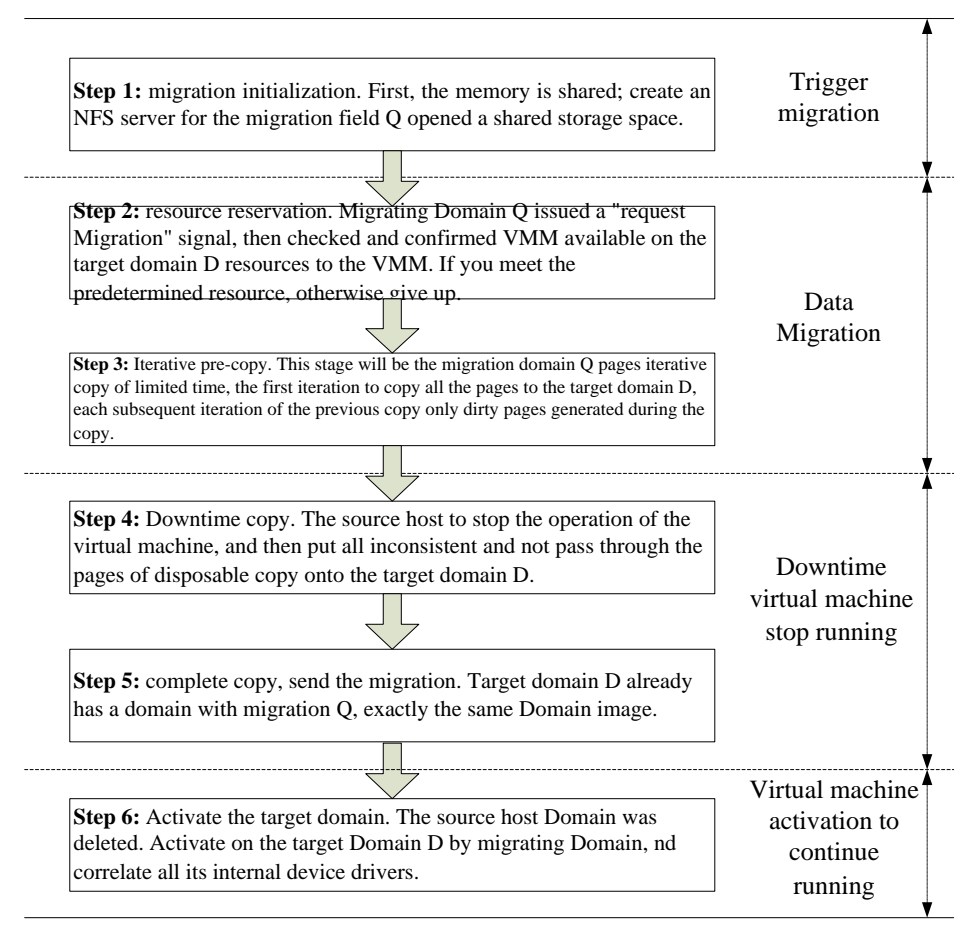

Fig.3: migration steps

The method of Figure 3 ensured that the migrated virtual machine at least be able to complete its mirror image is saved in the training of a cloud cluster hosts. Even if the migration fails, migrated objects will be re-activated, reassess the migration again, and protect the integrity of the system migration.

\section{Optimal Design of dynamic migration.}

Downtime and Migration time is often used as a performance evaluation of virtual machine live migration. Downtime mainly includes a virtual machine pauses, transfer CPU, and memory status, virtual machine recovery execution time, refers to the execution of virtual machine host at the source end from suspended beginning, to the virtual machine on the target host to resume execution of the period. It reflects the dynamic migration of virtual machines caused by system applications and service disruption time. Through the analysis of the whole process for dynamic migration, the main factors affecting the system performance is downtime. Because downtime will cause the virtual machine and the I/O devices such as interrupts, which led to the decrease of the I/O service quality or discontinuity. Therefore, reducing downtime, can effectively improve the virtual machine live migration performance. Dynamic migration framework with reference to FIG. 2, after re-design optimized framework as shown below: 


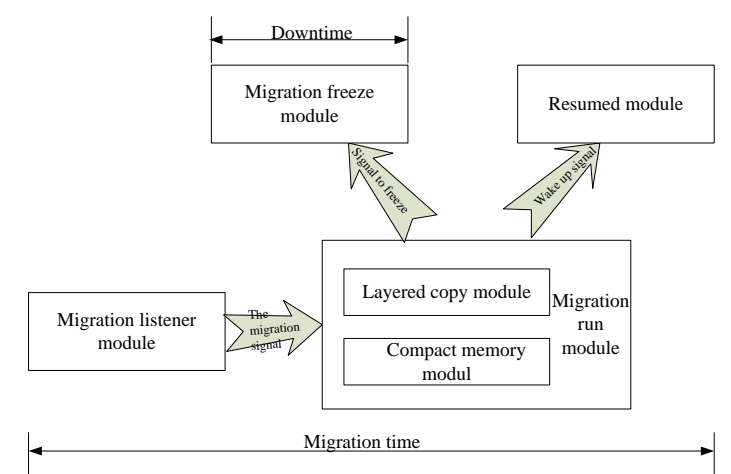

Fig.4: the optimal framework of VM dynamic migration

After optimization, the framework adds two sub-modules in the migration run the module: memory compression module and layered copy module. Memory compression module with the method of compression memory page migration domain, by reducing the amount of data required for migration and reduces network load, thereby enhancing the performance of dynamic migration; Layered copy module is based on the migration domain pages change frequency statistics, he domain pages were stratified according to the level of activity, first send less active part of the memory page, and then the active part of the memory pages one-time transfer, so that the transfer to reduce the workload within the downtime. Among them, migration module structure optimized is as follows:

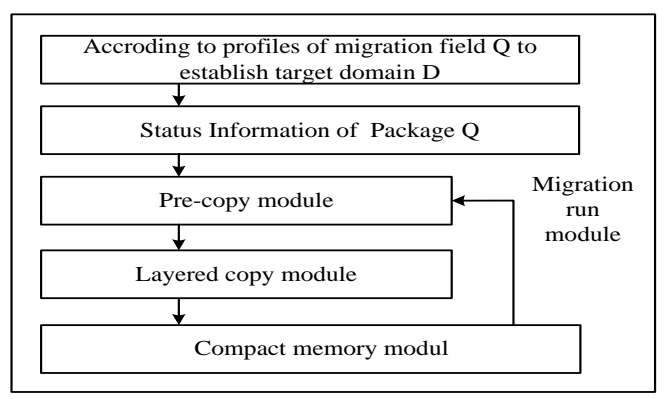

Fig.5: Migration Run Module

In figure 5, advance copy of the module function is to analyze the memory page running situation in advance, advance the statistics page memory activity, may be two situations: if it is detected that there is a large number (over 50\%) the memory page has been modified, it indicates that the current memory is high active, system into high dirty page rate environment, you need to call a layered copy module; if it detects only a small amount of memory pages are modified, indicates that the current memory activity is low, the system enters the dirty page rate environment, you also run using traditional migration module. The above optimization measures can effectively reduce the total migration time and downtime, reduce network load, maximizing the impact of reduced mobility caused by the service quality domain.

\section{Summary}

The use of practical cloud virtualization technology can significantly reduce equipment costs, get the higher reliability in data protection and disaster recovery, etc., but also conducive to the unified deployment, improve mobility and achieve low-carbon energy. This article as a starting point, on the basis of building a virtualized computing resources pool model, study how to implement the dynamic migration of virtual machines in a virtualized environment, so as to solve the problem of load balancing system. Hoping to provide some intentional and ideas for future research to explore cloud-related training technologies.

Fund Project: Nanjing Communications Institute of Technology research projects, Item Number: JY1202. 


\section{References}

[1] guang-bo Wang, etc. Cloud environment for load balancing of distributed virtual machine migration study [J].Computer applications and software, 10 ,pp. 87-91,2013.

[2] Pengcheng Liu. Research on cloud virtual machine live migration [D]. Shanghai: Fudan University, 2009.

[3] Daogong Tang. Under the environment of cloud resource scheduling strategy based on the virtual machine live migration research [D]. Chongqing: Chongqing University of posts and telecommunications, 2012 (in Chinese)

[4] Junke An. Application virtualization technology in the network construction Universities [J]. Information and Computer, 6 ,pp.97-99, 2011.

[5] H. Frank Cervone.An overview of virtual and cloud computing[J]. OCLC Systems \&Services ,Vol. 26 No. 3,pp.162-165,2010.

[6] Peng Liu. Cloud computing [M] Beijing: Electronic Industry Press, 2011: 1-132.

[7] Jinjun Liu etc. Based on the load characteristics of virtual machine migration scheduling strategy [J]. Computer Engineering,37 (17),pp.276-278, 2011.

[8] Yuanyuan Liu etc. The virtual machine resource load balancing methods under the Virtual Computing Environment [J]. Computer Engineering,36(16),pp. 30-32, 2010. 